\section{ORIGINAL RESEARCH}

\author{
D.-S. Yoo \\ Y.-D. Won \\ P.-W. Huh \\ H.-E. Shin \\ K.-T. Kim \\ S.-G. Kang \\ S.-B. Lee \\ K.-S. Cho
}

\title{
Therapeutic Results of Intra-Arterial Thrombolysis after Full-Dose Intravenous Tissue Plasminogen Activator Administration
}

\begin{abstract}
BACKGROUND AND PURPOSE: IV administration of tPA is accepted as a standard treatment for acute cerebral ischemia, but the clinical outcomes cannot be guaranteed in patients who are not recanalized after IV-tPA and in those who are not eligible for IV-tPA. In this study, outcomes from groups of patients treated with additional IA thrombolytic therapy with the use or omission of IV-tPA administration were compared.
\end{abstract}

\begin{abstract}
MATERIALS AND METHODS: IA thrombolytic therapy (thrombolytic agents combined with mechanical intervention) was attempted in those patients who were not eligible for IV-tPA and who showed continuous major vessel occlusion after IV-tPA. Sixty-three patients were divided into 2 groups: a tPA group ( $n=29$, IA thrombolysis after IV-tPA) and a non-tPA group ( $n=34$, IA thrombolysis without IV-tPA). These groups were subdivided according to match or mismatch DWI/PWI after MR imaging. Treatment results were compared by recanalization rate, clinical outcome, mortality, and ICH rate.
\end{abstract}

RESULTS: The recanalization rate was $79.3 \%$ in the tPA group and $55.9 \%$ in the non-tPA group ( $\chi^{2}$ test, $P<$.05). Subgroup analysis between DWI/PWI mismatch in the tPA group and DWI/PWI mismatch in the non-tPA group also showed no statistical difference in recanalization rate, favorable clinical outcome, and mortality ( $\chi^{2}$ test, $P>.05$ ), but the significant ICH rate was high in the tPA group ( $\chi^{2}$ test, $P<.05)$.

CONCLUSIONS: Additional IA thrombolytic treatment after full-dose IV-tPA administration might be an acceptable treatment option for patients with DWI/PWI mismatch.

\begin{abstract}
ABBREVIATIONS: Angio = angiography; $\mathrm{D} / \mathrm{P}=$ diffusion/perfusion; $\mathrm{DWI}=$ diffusion-weighted imaging; EVD = extra-ventricular drainage; GCS = Glasgow Coma Scale; GP $\mathrm{llb} / \mathrm{lla}=$ platelet glycoprotein receptor IIb/lla; IA = intra-arterial; ICA = internal carotid artery; ICH = intracerebral hemorrhage; IICP = increased intracranial pressure; IV = intravenous; Lt. = left; $\mathrm{MCA}=$ middle cerebral artery; MRI = MR imaging; $\mathrm{mRS}=$ modified Rankin Scale; NIHSS = National Institutes of Health Stroke Scale; NINDS = National Institute of Neurological Disorders and Stroke; PWI = perfusion-weighted imaging; Rt. = right; Sx. = symptom; TIMI = Thrombolysis in Myocardial Infarction; tPA = tissue plasminogen activator; Tx. = treatment
\end{abstract}

D espite the considerable advances in medicine, acute cerebral ischemia still produces a high fatality rate. ${ }^{1} \mathrm{IV}$ administration of tPA within 3 hours from the onset of ictus significantly reduces stroke severity and improves long-term clinical outcome. ${ }^{2}$ Treatment guidelines concerning IV-tPA prohibit the use of other thrombolytics 24 hours after tPA administration. ${ }^{3,4}$ However, IV-tPA is thought to be ineffective for the treatment of patients with occlusion of larger vessels, and a significant proportion of patients treated with IV-tPA show persistent cerebral vascular occlusion. Their prognosis depends on good fortune rather than therapeutic intervention. ${ }^{5-7}$ Several studies have demonstrated that IA delivery of thrombolytic agents allows greater diagnostic precision and

Received October 25, 2009; accepted after revision February 9, 2010.

From the Departments of Neurosurgery (D.-S.Y., P.-W.H.), Radiology (Y.-D.W., K.-T.K., S.-G.K., S.-B.L., K.-S.C.), and Neurology (H.-E.S.), Uijongbu St. Mary's Hospital, The Catholic University of Korea, College of Medicine, Uijeongbu, South Korea.

This work was supported by the Catholic Institute of Cell Therapy Basic Science Programs Foundation in the program year of 2008.

Please address correspondence to Do-Sung Yoo, MD, PhD, Department of Neurosurgery, Uijeongbu St. Mary's Hospital, 65-1, Kumoh-dong, Uijeongbu, 480-130, South Korea; e-mail: yooman@catholic.ac.kr

Indicates article with supplemental on-line table.

DOI 10.3174/ajnr.A2084 effective recanalization of the occluded cerebral vessel, with lessened stroke severity and improved outcome instead of complication risks. ${ }^{5-9}$ When possible, some studies performed combined IV/IA treatment, which is the concept of combining the advantages of IV-tPA (speed of and certainty of initiation of therapy as well as widespread availability) and IA recanalization therapy (titrated dosing, mechanical aids to recanalization, and possible superior and earlier recanalization). ${ }^{10-13}$ Although the results of IA or combined thrombolytic trials or both are encouraging, severe complications from treatment must be balanced against potential benefits. ${ }^{14}$

A recent study described the efficacy of using IV and/or IA thrombolysis tailored to the physiologic state of individual tissue and stratification of patients according to the potential risks of the therapies. ${ }^{15}$ The role of neuroimaging has become increasingly important in evaluating the extent and location of tissue at risk of infarction (ischemic penumbra) and to distinguish the vulnerable tissue from unsalvageable infarcted tissue or doomed hemorrhagic parenchyma. ${ }^{1,14-17}$ Nonenhanced CT of the brain is still the diagnostic standard for patients with acute cerebral ischemia, but advancements in neuroimaging techniques, especially DWI/PWI mismatch after MR imaging, may be exploitable. ${ }^{1,14-21}$

In this study, patients with acute cerebral ischemia were 


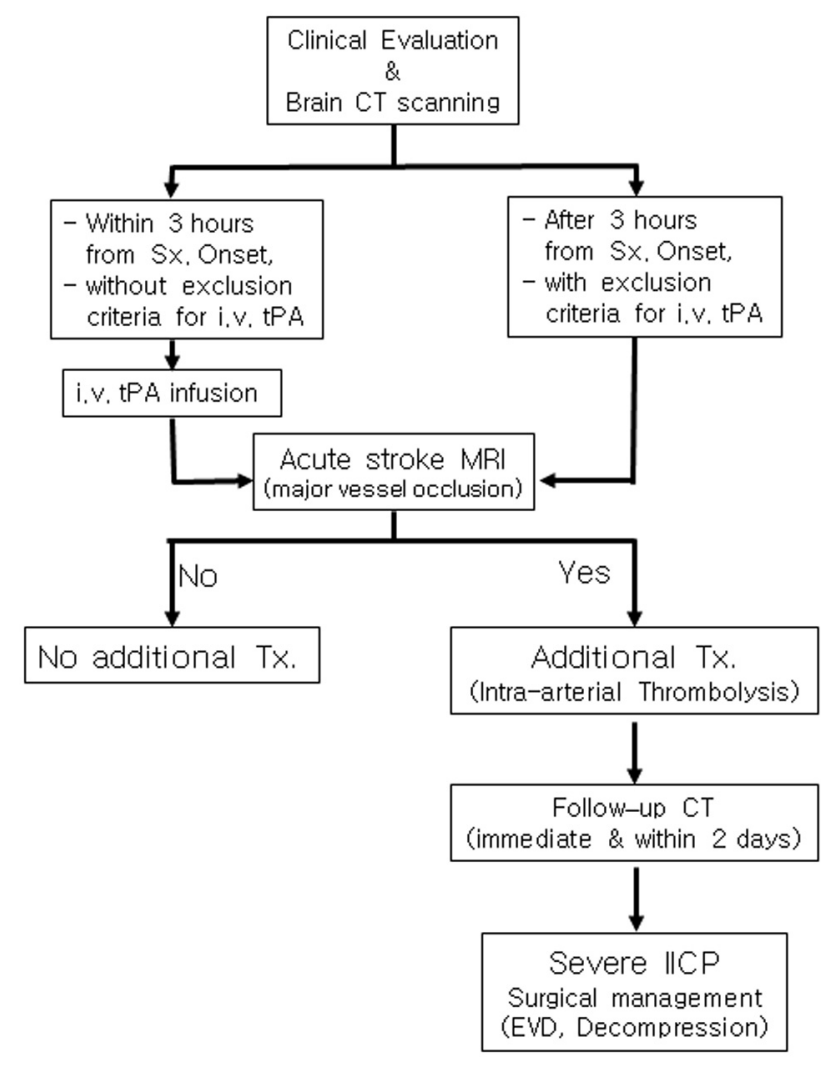

Fig 1. Flow diagram of the treatment protocol.

treated with IV-tPA when they presented within 3 hours from the onset of symptoms. Immediately thereafter, acute stroke MR imaging was conducted and additional IA thrombolytic therapy (thrombolytic agents combined with mechanical intervention) was used for those patients who were not eligible for IV-tPA or who presented with continuous major vessel occlusion after IV-tPA. We analyzed the hemorrhagic complication rates of additional IA thrombolytic therapy with the use or omission of IV-tPA and correlations between the clinical outcomes and DWI/PWI mismatch after MR imaging in the acute setting.

\section{Materials and Methods}

The treatment protocol (Fig 1) was approved by our institutional review board. All patients or their representatives signed informed written consent and were made aware that they were receiving an additional nonstandard therapy after IV-tPA therapy.

\section{Patient Selection and Neurologic Evaluation}

Between October 2004 and September 2008, 63 patients who had undertaken additional IA thrombolytic therapy due to anterior circulation major cerebral infarction were analyzed retrospectively. Demographic information, patient age, sex, radiologic findings, and clinical status are presented in On-line Table 1. The patients were divided into 2 groups. The tPA group comprised 29 patients who arrived at the hospital within 3 hours after symptom onset and who appropriately received IV-tPA. They had additional IA thrombolytic therapy for the major vessel occlusion even after IV-tPA. The non-tPA group consisted of 34 patients who arrived at the hospital 3-6 hours after symptom onset and who received only IA thrombolytic therapy without IV-tPA. Patients with exclusion criteria for IV-tPA were not treated with IA thrombolytic therapy. Treatment results were compared in terms of recanalization rate, clinical outcome, mortality, and clinically significant $\mathrm{ICH}$. The 2 groups were subdivided according to the DWI/PWI match $(\mathrm{DWI} / \mathrm{PWI} \leq 0.7)$ or mismatch $(\mathrm{DWI} / \mathrm{PWI}>0.7)$ based on MR imaging examination. These subgroup analyses compared the recanalization rate, favorable clinical outcome, mortality, and significant ICH between DWI/PWI mismatch in the tPA group $(n=23)$ and DWI/PWI mismatch in the non-tPA group $(n=24)$.

All patients underwent $\mathrm{CT}$ of the brain immediately after arrival at the emergency department. Patients with NIHSS scores $>8,18-75$ years of age, with no clinical improvement at the time of evaluation, were included in IV-tPA therapy following NINDS protocol. ${ }^{2} \mathrm{~Pa}$ tients meeting the inclusion criteria received IV-tPA $(0.9 \mathrm{mg} / \mathrm{Kg}$, initial $10 \%$ bolus infusion with the remainder infused during the next 60 minutes ${ }^{2}$ ). Patients who arrived 3-6 hours following symptom onset received only IA thrombolytic therapy. Initial neurologic status was measured by NIHSS scores at the time of arrival. mRS scores were measured 90 days after the onset of ischemia; scores of $0-1$ were considered a favorable outcome. An examiner blinded to the treatment recorded the scores. Overall mortality and complication rates were also recorded.

\section{Neuroradiologic Evaluations}

The neuroradiologic results were analyzed retrospectively by the neuroradiologist who had not performed the IA thrombolysis procedures. Immediately on completion of IV-tPA, an acute stroke MR imaging was performed, or in the cases in which no IV-tPA was administered, acute stroke MR imaging was performed following completion of the initial brain CT scan obtained by using a Somatom Sensation 16 scanner (Siemens, Malvern, Pennsylvania). The examinations used a clinical $1.5 \mathrm{~T}$ whole-body imager with a $24-\mathrm{mT} / \mathrm{m}$ gradient strength, a 300- $\mu$ s rise, and an echo-planar-capable receiver (Avanto; Siemens, Erlangen, Germany). Obtained images included T1-weighted sagittal scans, T2-weighted turbo-gradient and spinecho/echo-planar diffusion-weighted axial images, and perfusionweighted axial time-to-peak maps. Both DWI and PWI techniques have been described elsewhere. ${ }^{6,16}$ The total imaging and processing time was approximately 30 minutes. DWI data were automatically postprocessed to yield trace images from primary data at each section position. The PWI sequence generated a time-to-peak map for each section position. Areas of high signal intensity on DWIs and areas of decreased perfusion on time-to-peak maps for PWIs were used to calculate the mismatch ratio. ${ }^{1,14,16,22}$ After IA thrombolytic therapy, all patients underwent brain CT scanning immediately and again within 2 days. Non-contrast-enhanced brain CT was performed after IA thrombolytic therapy, and recheck brain CT angiography was performed within 2 days. Increased attenuation on both follow-up CT scans could reflect extravasation of the contrast media, hemorrhage, or both. ${ }^{23}$ "Symptomatic ICH" was defined as neurologic worsening of $>4$ points in the NIHSS score that was attributable to the presence of clot. "Reperfusion injury" was defined as massive brain swelling or hemorrhage while the occluded vessel was recanalized.

\section{IA Thrombolysis}

If there was major vessel occlusion on MR imaging of acute stroke, cerebral angiography and additional IA thrombolytic therapy (combined thrombolytics with mechanical intervention) were tried. Angiograms were obtained by using standard angiographic techniques. A $6 \mathrm{~F}$ sheath was generally introduced into the right femoral artery. 
Diagnostic angiograms were obtained in all intracranial vessels by using $5 \mathrm{~F}$ diagnostic catheters. Once an occlusion was noted, the diagnostic catheter was exchanged for a $6 \mathrm{~F}$ guiding catheter, which was placed into the ICA. A 2.3F end-hole microcatheter was typically used and guided to the occluded vessel over a microguidewire. The tip of the microcatheter was typically embedded in the thrombus. Thrombolytic agents usually were infused through a microinjection pump. Urokinase and abciximab (ReoPro) (GP IIb/IIIa antagonist) were the thrombolytics of choice. The initial dose of urokinase was 200,000 U (diluted with 10,000 U/mL of saline) administered close to the clot for 10 minutes. Repeated doses were administered until the planned doses (urokinase, 500,000 U, and ReoPro, $10 \mathrm{mg}$ ) were achieved. In all instances, mechanical disruption was also attempted. Heparin was used with a bolus dose of $3000 \mathrm{U}$ at the beginning of the angiography. The end point for thrombolytic infusions was complete recanalization or attainment of the maximum planned dose. Recanalization was considered failed (TIMI grade 0 or 1 ) or successful (TIMI grade 2 or 3). In some cases, delayed recanalization occurred; presently, it is defined as "failed." A follow-up nonenhanced CT scan was obtained just after IA thrombolytic therapy, and a CT angiography was performed within 2 days after the treatment.

\section{Additional Surgical Intervention}

Decompressive craniectomy was performed in case of increased intracranial pressure due to massive brain edema or $\mathrm{ICH}$, with the following indications: midline shift of $>6 \mathrm{~mm}$ with effaced cortical sulci and cisternal structures, with neurologic deterioration ( $>2$-point worsening of GCS or $<8$ points of GCS). ${ }^{24}$

\section{Statistical Analyses}

All data are presented as the mean \pm SD or as the median. A Wilcoxon signed rank sum test was used to analyze NIHSS scores. Comparisons among groups were computed by using the unpaired $t$ test and Fisher exact test. Statistical significance was defined as a $P$ value $\leq .05$.

\section{Results}

\section{Comparison of tPA and Non-tPA Groups}

Recanalization rate and mortality between the $\mathrm{PPA}(79.3 \%$ and $3.45 \%$, respectively) and non-tPA groups (55.9\% and $11.8 \%$, respectively) showed no statistical differences ( $\chi^{2}$ test, $\left.P>.05\right)$ (On-line Table 1). Favorable clinical outcomes based on the mRS score were obtained in $34.5 \%$ of the tPA group and $44.1 \%$ of the non-tPA group $\left(\chi^{2}\right.$ test, $\left.P>.05\right)$. Significant ICH occurred in 6 patients $(20.7 \%)$ in the tPA group and in 4 patients $(11.8 \%)$ in the non-tPA group $\left(\chi^{2}\right.$ test, $\left.P>.05\right)$. In subgroups, the analysis showed that significant ICH occurred in $17.4 \%$ of the mismatch tPA group but in $0 \%$ of the mismatch non-tPA group $\chi^{2}$ test, $P<.05$ ).

\section{Comparison of the DWI/PWI Match and Mismatch Groups}

Analysis according to the DWI/PWI ratio regardless of IV-tPA administration revealed a recanalization rate and favorable outcome of $50.0 \%$ and $6.3 \%$, respectively, in the match group and $72.3 \%$ and $53.2 \%$, respectively, in the mismatch group $\left(\chi^{2}\right.$ test, $P<.05)$. The mortality and significant hemorrhagic complication rate were $31.3 \%$ and $37.5 \%$, respectively, in the match group and $0 \%$ and $8.5 \%$, respectively, in the mismatch group ( $\chi^{2}$ test, $\left.P<.05\right)$ (On-line Table 1$)$. The match group showed a higher incidence of significant hemorrhagic complication rate and mortality than the mismatch group.

\section{Comparison of DWI/PWI Mismatch tPA and Non-tPA Groups}

Comparison of the DWI/PWI mismatch tPA and non-tPA groups showed similar results in recanalization rate, favorable clinical outcome, and mortality $(78.3 \%, 43.5 \%$, and $0 \%$, respectively for the tPA group versus $66.7 \%, 58.3 \%$, and $0 \%$, respectively, for the non-tPA group). However, a significant hemorrhagic complication rate was more frequent in the mismatch tPA than in the mismatch non-tPA group $\left(\chi^{2}\right.$ test, $P<$ $.05)$.

\section{Other Findings}

In brain CT conducted after additional IA thrombolytic therapy, extravasation of the contrast media was the most frequent finding (17 cases, 27\%). The initial neurologic status and end clinical outcomes according to laterality showed no statistical difference both in the MCAs and ICAs, respectively ( $\chi^{2}$ test, $P>$.05). Decompressive craniectomy was performed in 6 patients in the tPA group and in 3 patients in non-tPA group; and in all cases, the clinical outcome was grave.

\section{Discussion}

Thrombolytic stroke therapy is based on the ischemic penumbra. ${ }^{17,25-27}$ According to the recanalization hypothesis, reopening of occluded vessels before critical cell injury might improve clinical outcome in acute ischemic stroke through regional reperfusion and salvage of threatened tissue. ${ }^{1,17,18,25,28}$ Early vessel recanalization is linked powerfully to final clinical outcome in acute ischemic stroke. ${ }^{28,29}$

Even though IV-tPA is approved by the US Food and Drug Administration and the American Stroke Association has recommended this treatment as a standard in patients with stroke, the recanalization rate of IV-tPA therapy is questionable. ${ }^{3}$ Indeed, the treatment has been criticized as being ineffective for patients with occlusion of large vessels such as the MCA and ICA and there are no recommended additional treatment guidelines for cases of nonrecanalization after IV-tPA. ${ }^{3-5,29}$

IA treatment permits an accurate diagnosis and can facilitate the application of a higher concentration of the thrombolytic agent in the vicinity of the clot followed by mechanical destruction of the clot. ${ }^{7}$ In addition to these advantages of IA thrombolysis, the increased percentage of recanalization, which ranges from $45.5 \%$ to $94 \%$, can extend the therapeutic time window by as much as 6 hours from the onset of acute ischemic stroke. ${ }^{1,3,5,8,29-31}$ It has been suggested that recanalization might have a deleterious influence on the hemorrhagic conversion of the infarct, but analysis of pooled data failed to demonstrate an increased rate of hemorrhagic transformation in patients experiencing recanalization. ${ }^{29,32}$

In our study, extravasation of the contrast media was the most frequent finding, and increased incidence of extravasation correlated with increased incidence of significant ICH. Well-reviewed reports concerning the thrombolytic dose and agents have concluded that when more thrombolytics were used, more hemorrhagic complications occurred. ${ }^{33}$ Symptomatic ICH occurred in $20.7 \%$ of the tPA group and $11.8 \%$ of 
the non-tPA group. However, in subgroup analysis, DWI/ PWI mismatch subgroups showed a symptomatic ICH of $8.5 \%$, while it was $37.5 \%$ in the DWI/PWI match groups $\left(\chi^{2}\right.$ test, $P<.05)$. Moreover, the analysis of DWI/PWI mismatch subgroups showed that symptomatic ICH occurred in $17.4 \%$ of the tPA group but not in the non-tPA group $\left(\chi^{2}\right.$ test, $P<$ $.05)$. From this study, we confirmed that a larger amount of thrombolytic agents results a greater incidence of symptomatic ICH. Although the symptomatic hemorrhagic rate in the tPA group was high compared with NINDS Trial results (6.4\%), mortality was low $(3.5 \%$ in the tPA group and $11.8 \%$ in the non-tPA group versus $17 \%$ in the NINDS Trial) and favorable outcome was high $34.5 \%$ in the tPA group and $44.1 \%$ in the non-tPA group versus $26 \%$ in the NINDS Trial). ${ }^{2,5,8,32,34}$ However, the symptomatic hemorrhagic rate in the non-tPA group was similar to rates found in other IA thrombolysis trials. ${ }^{8,31}$

The guidelines of the American Stroke Association do not recommend combined IA and IV therapy, ${ }^{4}$ but previous reports concerning acute coronary syndrome and acute cerebral ischemia point out some advantages of the combined therapy, which include significant reduction in the total amount of thrombolytic drug required, easy conversion to mechanical thrombolysis, and the safety of ReoPro in acute ischemic stroke, even with a full dosage for acute coronary syndrome. ${ }^{5,6,30,34-36}$

Some previous studies performed the combined IV/IA therapy in a prospective manner, evaluating IA therapy for acute stroke following IV-tPA administration. In these studies, IV-tPA doses were at the reduced level of $0.6 \mathrm{mg} / \mathrm{kg}$ and the remaining dose of tPA was administered via the arterial route. In the Emergency Management of Stroke Bridging Trial, ${ }^{11}$ there were trends toward a higher ICH rate, higher mortality, and poorer clinical outcomes in the combined IV/IA therapy group. However, in Interventional Management of Stroke I and II, the combined IV/IA therapy results showed trends toward better clinical outcomes than the IV-tPA-treated group in the NINDS Trial. In our study, the combination of thrombolytics for the nonrecanalized patients after IV-tPA administration was effective in improving patient outcome. Even though the complication rate increased, the clinical outcome was beneficial.

In our study, the mortality rate was definitely lower than that in previous studies. Some authors thought that timely performance of surgical intervention for increased intracranial pressure critically decreased the mortality rate. ${ }^{24}$

Conventional noncontrast CT remains the mainstay in the imaging of suspected acute stroke. ${ }^{1,12}$ However, MR imaging has emerged as an important tool for the identification of patients with cerebral ischemia. ${ }^{1,6,14,15,18-21}$ Methods for evaluating blood flow and tissue viability are increasingly required because therapeutic interventions may be tailored to the physiologic state of the individual tissue for the stratification of patients according to the potential risks of therapies. ${ }^{1,14,17,37}$ The usefulness of MR imaging has been enhanced with the combination of DWI and PWI to reveal cerebral infarctions and ischemia among patients with early acute ischemic strokes. During this acute period, abnormal PWI areas can be larger than the DWI areas. These PWI/DWI mismatches may represent tissue at risk for cerebral infarction unless blood flow is restored. ${ }^{1,14,15,17,19,20}$ It is not easy to calculate the accurate DWI/PWI mismatch rate. Standard perfusion images can be variable, and a standard image can be difficult to determine. ${ }^{1,22}$ Nonetheless, these techniques are feasible and applicable before therapy for patients with acute strokes, permitting immediate triage and avoiding potential risks of cerebral angiography and thrombolytic therapy. ${ }^{5,6,16,18,37}$

In this study, the DWI/PWI match group had unacceptable clinical results. However, in the DWI/PWI mismatch group, recanalization rate, favorable clinical outcome, and significant hemorrhagic rate were similar to those found in a previous IA thrombolysis trial undertaken within 3 hours after symptom onset. ${ }^{5}$ Our present comparison of the DWI/PWI mismatch tPA and non-tPA groups did not reveal statistically significant differences in favorable outcome, mortality, and significant hemorrhagic complication rate. In our study, the mismatch tPA group showed a higher incidence of favorable outcome $(43.5 \%)$ than that in the NINDS Trial (39\%). ${ }^{2}$ This mismatch tPA patient group might have had an unfavorable outcome if these patients had not been treated with additional IA thrombolytic therapy. For patients who fail recanalization after IVtPA, IA thrombolysis can be attempted, especially in patients with DWI/PWI mismatch.

Our report has limitations, which include the lack of a control group, small sample size, and patient selection. Nevertheless, this report suggests that additional IA thrombolytic therapy for selected patients, such as those with MR imagingevident DWI/PWI mismatch after IV-tPA or those not eligible for IV-tPA, improves the clinical outcome.

\section{Conclusions}

Patients with DWI/PWI mismatch on acute stroke MR imaging show a higher recanalization rate, better clinical outcome, and a lower hemorrhagic complication rate. Additional IA thrombolytic therapy after a full dose of IV-tPA, in the case of nonrecanalization, increases the favorable outcome rate and decreases mortality. Careful application of the additional IA thrombolytic therapy according to DWI/PWI mismatch may improve outcomes in patients at the end of treatment.

\section{References}

1. Abe O, Aoki S, Shirouzu I, et al. MR imaging of ischemic penumbra. Eur J Radiol 2003;46:67-78

2. Tissue plasminogen activator for acute ischemic stroke: The National Institute of Neurological Disorders and Stroke rt-PA Stroke Study Group. New Engl J Med 1995;333:1581-87

3. Hacke W, Kaste M, Fieschi C, et al, for the ECASS Study Group. Intravenous thrombolysis with recombinant tissue plasminogen activator for acute hemispheric stroke: The European Cooperative Acute Stroke Study (ECASS). JAMA 1995;274:1017-25

4. Adams HP Jr, del Zoppo G, Alberts MJ, et al. Guidelines for the early management of adults with ischemic stroke: a guideline from the American Heart Association/American Stroke Association Stroke Council, Clinical Cardiology Council, Cardiovascular Radiology and Intervention Council, and the Atherosclerotic Peripheral Vascular Disease and Quality of Care Outcomes in Research Interdisciplinary Working Groups-the American Academy of Neurology affirms the value of this guideline as an educational tool for neurologists. Stroke 2007;38:1655-711. Epub 2007 Apr 12

5. Bourekas EC, Slivka AP, Shah R, et al. Intraarterial thrombolytic therapy within 3 hours of the onset of stroke. Neurosurgery 2004;54:39-46

6. Suarez JI, Zaidat OO, Sunshine JL, et al. Endovascular administration after intravenous infusion of thrombolytic agents for the treatment of patients with acute ischemic strokes. Neurosurgery 2002;50:251-60

7. Madden K. Optimal timing of thrombolytic therapy in acute ischaemic stroke. CNS Drugs 2002;16:213-18

8. Kase CS, Furlan AJ, Wechsler LR, et al. Cerebral hemorrhage after intra- 
arterial thrombolysis for ischemic stroke: the PROACT II trial. Neurology 2001;57:1603-10

9. Macleod MR, Davis SM, Mitchell PJ, et al. Results of a multicentre, randomised controlled trial of intra-arterial urokinase in the treatment of acute posterior circulation ischaemic stroke. Cerebrovasc Dis 2005;20:12-17. Epub 2005 May 30

10. Mazighi M, Serfaty JM, Labreuche J, et al, for the RECANALISE investigators. Comparison of intravenous alteplase with a combined intravenousendovascular approach in patients with stroke and confirmed arterial occlusion (RECANALISE study): a prospective cohort study. Lancet Neurol 2009;8: 802-09. Epub 2009 Jul 31

11. Lewandowski CA, Frankel M, Tomsick TA, et al. Combined intravenous and intra-arterial r-TPA versus intra-arterial therapy of acute ischemic stroke: Emergency Management of Stroke (EMS) Bridging Trial. Stroke 1999;30: $2598-605$

12. IMS Study Investigators. Combined intravenous and intra-arterial recanalization for acute ischemic stroke: the Interventional Management of Stroke Study. Stroke 2004;35:904-11. Epub 2004 Mar 11

13. IMS II Trial Investigators. The Interventional Management of Stroke (IMS) II Study. Stroke 2007;38:2127-35. Epub 2007 May 24

14. Grandin CB. Assessment of brain perfusion with MRI: methodology and application to acute stroke. Neuroradiology 2003;45:755-66

15. Albers GW, Thijs VN, Wechsler L, et al, for the DEFUSE investigators. Magnetic resonance imaging profiles predict clinical response to early reperfusion: the diffusion and perfusion imaging evaluation for understanding stroke evolution (DEFUSE) study. Ann Neurol 2006;60:508-17

16. Singer OC, Du Mesnil De Rochemont R, Foerch C, et al. Early functional recovery and the fate of the diffusion/perfusion mismatch in patients with proximal middle cerebral artery occlusion. Cerebrovasc Dis 2004;17:13-20. Epub 2003 Oct 3

17. Masdeu JC, Irimia P, Asenbaum S, et al. EFNS guideline on neuroimaging in acute stroke: report of an EFNS task force. Eur J Neurol 2006;13:1271-83

18. Schellinger PD, Jansen O, Fiebach JB, et al. Monitoring intravenous recombinant tissue plasminogen activator thrombolysis for acute ischemic stroke with diffusion and perfusion MRI. Stroke 2000;31:1318-28

19. Schellinger PD, Thomalla G, Fiehler J, et al. MRI-based and CT-based thrombolytic therapy in acute stroke within and beyond established time windows: an analysis of 1210 patients. Stroke 2007;38:2640-45

20. Kane I, Sandercock P, Wardlaw J. Magnetic resonance perfusion diffusion mismatch and thrombolysis in acute ischaemic stroke: a systematic review of the evidence to date. J Neurol Neurosurg Psychiatry 2007;78:485-90

21. Davis SM, Donnan GA, Parsons MW, et al, for the EPITHET investigators. Effects of alteplase beyond $3 \mathrm{~h}$ after stroke in the Echoplanar Imaging Thrombolytic Evaluation Trial (EPITHET): a placebo-controlled randomised trial. Lancet Neurol 2008;7:299-309. Epub 2008 Feb 28

22. Grandin CB, Duprez TP, Smith AM, et al. Which MR-derived perfusion parameters are the best predictors of infarct growth in hyperacute stroke? Com- parative study between relative and quantitative measurements. Radiology 2002;223:361-70

23. Yoon W, Seo JJ, Kim JK, et al. Contrast enhancement and contrast extravasation on computed tomography after intra-arterial thrombolysis in patients with acute ischemic stroke. Stroke 2004;35:876-81

24. Kim KT, Park JK, Kang SG, et al. Comparison of the effect of decompressive craniectomy on different neurosurgical diseases. Acta Neurochir (Wien) 2009; 151:21-30

25. Astrup J, Siesjö BK, Symon L. Thresholds in cerebral ischemia: the ischemic penumbra. Stroke 1981;12:723-25

26. Heiss WD, Grond $M$, Thiel A, et al. Tissue at risk of infarction rescued by early reperfusion: a positron emission tomography study in systemic recombinant tissue plasminogen activator thrombolysis of acute stroke. J Cereb Blood Flow Metab 1998;18:1298-307

27. Furlan M, Marchal G, Viader F, et al. Spontaneous neurological recovery after stroke and the fate of the ischemic penumbra. Ann Neurol 1997;41:411-14

28. Neumann-Haefelin T, du Mesnil de Rochemont R, Fiebach JB, et al, for the Kompetenznetz Schlaganfall Study Group. Effect of incomplete (spontaneous and postthrombolytic) recanalization after middle cerebral artery occlusion: a magnetic resonance imaging study. Stroke 2004;35:109-14. Epub 2003 Dec 11

29. Rha JH, Saver JL. The impact of recanalization on ischemic stroke outcome: a meta-analysis. Stroke 2007;38:967-73

30. Lee DH, Jo KD, Kim HG, et al. Local intraarterial urokinase thrombolysis of acute ischemic stroke with or without intravenous abciximab: a pilot study. $J$ Vasc Interv Radiol 2002;13:769-74

31. Furlan A, Higashida R, Wechsler L, et al. Intra-arterial prourokinase for acute ischemic stroke: The PROACT II study - a randomized controlled trial. Prolyse in Acute Cerebral Thromboembolism. JAMA 1999;282:2003-11

32. Paciaroni M, Agnelli G, Corea F, et al. Early hemorrhagic transformation of brain infarction: rate, predictive factors, and influence on clinical outcomeresults of a prospective multicenter study. Stroke 2008;39:2249-56

33. Mielke O, Wardlaw J, Liu M. Thrombolysis (different doses, routes of administration and agents) for acute ischaemic stroke. Cochrane Database Syst Rev 2004;18:CD000514

34. Abou-Chebl A, Bajzer CT, Krieger DW, et al. Multimodal therapy for the treatment of severe ischemic stroke combining GPIIb/IIIa antagonists and angioplasty after failure of thrombolysis. Stroke 2005;36:2286-88. Epub 2005 Sep 22

35. Rashdan I, Schechter E. Safety and efficacy of abciximab use in conjunction with intracoronary urokinase in patients requiring angioplasty. Catheter Cardiovasc Interv 2000;49:113-16

36. Qureshi AI, Suri MF, Khan J, et al. Abciximab as an adjunct to high-risk carotid or vertebrobasilar angioplasty: preliminary experience. Neurosurgery 2000;46: 1316-24

37. Ringleb PA, Schellinger PD, Schranz C, et al. Thrombolytic therapy within $\mathbf{3}$ to 6 hours after onset of ischemic stroke: useful or harmful? Stroke 2002;33: 1437-41 